\title{
Poésie
}

\section{Rhoddy Attilus}

\section{Aimer}

Une faÃßon de mourir

Câ€ $€^{\mathrm{TM}}$ est dâ€ $€^{\mathrm{TM}}$ aimer

Une faÃßon dâ€TM exister $\tilde{A}$ tout jamais

Dâ€ ${ }^{\mathrm{TM}}$ arr $\tilde{A}^{a}$ ter le temps

De combler lâ€ $€^{\mathrm{TM}}$ espace

Câ $€^{\mathrm{TM}}$ est dâ€ $€^{\mathrm{TM}}$ aimer

Une faÃßon de comprendre

Que tout a un langage

Que tout a une conscience

Que ce soit ce caillou

PiÃ $\subseteq$ tin $\tilde{A} \subseteq$ au milieu de la route

Ce tourbillon de poussi $\tilde{A}^{\prime \prime}$ re

Qui trouble lâ $€^{\text {TM }}$ homme sur son chemin

Ou cette feuille tombÃ $(e)$ dans lâ€ $€^{\mathrm{TM}}$ eau

Qui ne veut pas mourir

Câ€ $€^{\mathrm{TM}}$ est dâ€ $€^{\mathrm{TM}}$ aimer

Une faÃßon de comprendre

Que tout est $d \tilde{A} @$ risoire

Que tout est insensÃ (

Sauf ce Ã quoi

Lâ€ $€^{\mathrm{TM}}$ amour a donnÃ $($ un sens

Câ€ $€^{\mathrm{TM}}$ est dâ€ $€^{\mathrm{TM}}$ aimer

Une faÃßon de voir lâ€ $€^{\mathrm{TM}}$ invisible

De sentir lâ€ $€^{\mathrm{TM}}$ inodore

De palper lâ€ $€^{\mathrm{TM}}$ intouchable

Dâ€ $\epsilon^{\mathrm{TM}}$ entendre lâ€ $\epsilon^{\mathrm{TM}}$ inaudible

Câ€ $€^{T M}$ est dâ€ $€^{T M}$ aimer

Une faÃßon de donner chair

$\tilde{A} €$ lâ€ $\epsilon^{\mathrm{TM}} \operatorname{esp} \tilde{A} @$ rance

Dâ€ $€^{\mathrm{TM}}$ apprivoiser la vie

Et dâ€ $€^{\mathrm{TM}} \tilde{A}$ atre libre

Câ€ ${ }^{\mathrm{TM}}$ est dâ€ $€^{\mathrm{TM}}$ aimer

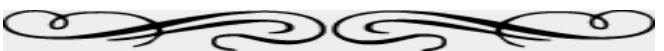

\section{Les grandes vÃ@ritÃ@s}

Tout ce que le feu touche

Devient feu

Tout ce que lâ€ $€^{\mathrm{TM}}$ amour touche

Devient amour

Lâ€ ${ }^{\text {TM }}$ homme

Touch $\tilde{A} @$ par la tendresse

Devient tendre 
Et le sourire

Est une maladie

Contagieuse.

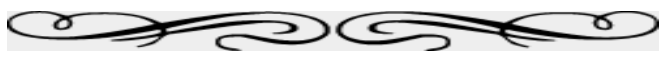

\section{Les insensÃ@s}

Nous sommes tous des riverains

Et la rivi $\tilde{A}^{\prime \prime}$ re peut monter

Aujourdâ $€^{\text {TM }}$ hui ou demain

Que deviendront nos vanitÃ $@$ s

Dans lâ€ $€^{\mathrm{TM}}$ eau qui les aura emportÃ@es

Nous les regarderons

Sâ€ $€^{\mathrm{TM}}$ enfuir

Loin de nous

Et nous saurons

Que nous avons $\tilde{A}(t) \tilde{A}($ insensÃ $(s$

Dâ€ ${ }^{\mathrm{TM}}$ avoir installÃ $\odot$ notre cÄur

Sur les seuls biens qui passent

Et nous saurons

Que nous avons $\tilde{A} @ t \tilde{A} \Subset$ imb $\mathrm{A} @$ ciles

De nous $\tilde{A}$ atre $\tilde{A} @$ tablis

Dans nos seuls conforts

MatÃ@riels

Et nous saurons enfin

Que les meilleures richesses

Dâ€ $\epsilon^{\mathrm{TM}}$ une vie

Sont celles

Que lâ€ $€^{\mathrm{TM}}$ or et lâ€ $€^{\mathrm{TM}}$ argent

Ne peuvent procurer.

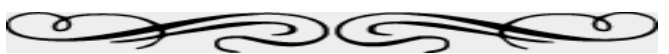

\section{Tite Fille}

Tite fille aux sentiers des montagnes

Nâ€ $\epsilon^{\mathrm{TM}}$ as-tu pas peur dâ€ $€^{\mathrm{TM}} \tilde{A}^{a}$ tre renvers $\tilde{A}(e$

Tu traÃ $\circledast$ nes des pantoufles

Sur les flancs des collines

Un lourd fardeau

PÃ "se sur ta tÃate

Tu tâ $€^{\mathrm{TM}}$ en vas

Sous le soleil et sous la pluie

Soleil ardent et pluie abondante

Trois heures six heures

Tu es en route

Avec ton gros sac sur ta tãate

Tu vois sâ€ $€^{\mathrm{TM}}$ en aller le soleil

Avec la fin de la jourñ̃ ()e

Tu continues Ã tâ $€^{\mathrm{TM}}$ en aller

$\operatorname{TrÃ} @$ buchante aveuglÃ $(e)$ 


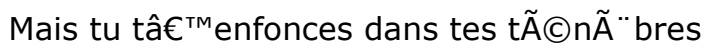

Qui envahissent ton existence

Tu ne verras point un autre jour

Cette nuit est soutenue par des traÃ $\circledast$ tres

Tite fille aux sentiers des montagnes

Adieu la vie

Salut la mort

Et dÃ $@$ sormais

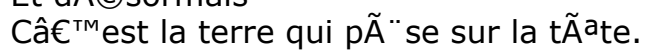

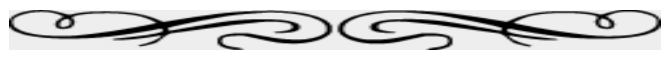

\section{Il est un pays}

Il est un pays

OA $\tilde{A}^{1}$ lâ€ $€^{\mathrm{TM}}$ on entend rien

Si des enfants ne pleurent pas

Si des poules

Sortant de leur nid

Ne caquettent pas

Ou si des rires

Ne pÃ@tillent pas

$\tilde{A} €$ chaque coin du bois

Pour quoi ce peuple rit-il

Si souvent

Pourquoi rit-il

Plus quâ€ $€^{\mathrm{TM}}$ il ne pleure

Mais il rÃ@pond

Mieux vaut $\tilde{A}$ atre laid

Mais vivant

Et la vie

MÃame vÃ@cue dans la crasse

Est prã@fÃ@rable

$\tilde{A} €$ la mort

Et nous rirons

Le long de nos jours

Fussent-ils sombres

Ou heureux

Dans ce pays

O $\tilde{A}^{1} \mid a ̂ €^{T M}$ on entend rien

On entend la mis $\tilde{A}^{*}$ re

MÃalÃ@e de tam-tam

Et de danse

Et de transe

On entend le cafÃ $\bigodot$

Le tafia

Tomber sur le sol

En mÃ@moire des morts

Dans ce pays

Silencieux

On entend aussi

On entend surtout

Lâ€ $€^{\mathrm{TM}}$ amour 
Bruire

Avec les arbres

Et lâ€ $€^{\mathrm{TM}}$ amour nous fait Ã@gaux

$\tilde{A} €$ tous les peuples

De lâ€ $€^{\mathrm{TM}}$ univers.

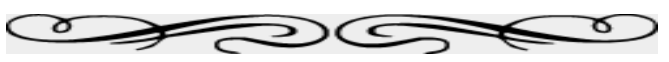

\section{Femme noire}

La nuit pose sur toi

Avec son calme

Et son silence

Et ses myst $\tilde{A}^{-1}$ res

Femme noire

Du ciel nocturne

Tombe la pÃ $@$ nombre

Serpentant ta silhouette

Mystique

EnvoÃ»tante

Et les contours sont ceux

Dâ $€^{\mathrm{TM}}$ une terre montagneuse

RavinÃ $@ e$

VallonnÃ (Ce

Profil de lâ€ $€^{\mathrm{TM}}$ immortalitÃ $\subset$

Ecriture avant toute $\tilde{A} @$ criture

Dans lâ€ $\epsilon^{\mathrm{TM}} \tilde{A}\left(\right.$ ternit $\tilde{A}\left({ }^{\circ}\right.$

De lâ€ $€^{\mathrm{TM}} A m \tilde{A} @$ rique prÃ@colombienne

TracÃ@ mystã $($ rieux

Sur le paroi de mon coeur

Au milieu de tes eaux dâ€ $€^{\mathrm{TM}}$ amour

De Samana

Femme dâ€ ${ }^{\mathrm{TM}}$ ombre

Femme sombre

La nuit sur ton corps

Câ€ $€^{\mathrm{TM}}$ est moi

Tu me berces

Tu mâ€ ${ }^{\mathrm{TM}}$ endors

Tu me fraies un chemin

Vers ta butte de Charrier

OÃ ${ }^{1}$ je livre

Lâ€ $€^{\mathrm{TM}}$ ultime combat

Dâ€ ${ }^{\mathrm{TM}}$ une conquÃate dâ€ $\epsilon^{\mathrm{TM}}$ amour

Embrasant

Ta vaste pÃ@nÃ@plaine du nord

Depuis les hauteurs

De ta buste

SacrÃ@e

Juste dans tes rÃ@gions reculÃ $@ e s$

De Joli trou

OÃ ${ }^{1}$ lâ€ $€^{\mathrm{TM}}$ incendie sâ€ $€^{\mathrm{TM}}$ attisera

Et mourra. 